\title{
Plicatura gástrica laparoscópica: seguimiento a largo plazo
}

\author{
Juan Eduardo Contreras-Parraguez ${ }^{1,2}$, Julio César Núñez-Villegas ${ }^{1,2}$, \\ Diva Villao-Maridueña ${ }^{1,2}$ y Jorge Bravo-López ${ }^{1,2}$
}

'Servicio de Cirugía, Hospita del Salvador. Santiago, Chile. ¿Departamento de Cirugía Oriente, Facultad de Medicin Universidad de Chile. Santiago, Chile.

Recibido 2020-07-28 aceptado 2020-08-2

Correspondencia a: Dr. Juan Eduardo ContrerasParraguez uaneduardocontreras@ gmail.com

\section{Laparoscopic gastric plication: long-term follow-up}

Background: The international experience has failed to reproduce the first studies of laparoscopic gastric plication (LGP). Aim: The objective is to analyze the outcomes after 10 years of follow-up of patients subjected to LGP. Materials and Method: Prospective and descriptive study, in which obese adult patients who met universal criteria for bariatric surgery were included. Epidemiological data, comorbidities, operating time, hospital stay, percentage of excess BMI loss (\% EBMIL) and resolution of comorbidities were collected. The follow-up was realized by annual periodic controls until 2020. Results: Patient selection began in 2010. A total of 26 interventions were performed from January 2011 to May 2012. All patients were female. The average preoperative Body Mass Index (BMI) was $38.8 \mathrm{~kg} / \mathrm{m}^{2}$ (SD 3.8). The average $\%$ EBMIL at $1^{\text {st }}, 3^{\text {rd }}$ and $9^{\text {th }}$ postoperative years was $62.2 \%$ (SD 27.1), 40.2\% (SD 24.5) and 28\% (SD 31.9), respectively. Complications, based in Clavien Dindo classification, during the first 30 postoperative days was: 21 patients with type I, 1 type II, and 2 patients with complications type IVa. At $9^{\text {th }}$ postoperative year, 9 patients presented adverse effect type I. There was no mortality. Until the $3^{\text {rd }}$ year there was correction of comorbidities. Three diabetic patients were observed at the end of the study, 2 previously healthy. Conclusions: The long term \% EBMIL was insufficient. The percentage of complications is higher than in other techniques. We do not recommend the LGP.

Key words: gastric plication; bariatric surgery; obesity.

\section{Resumen}

Introducción: La experiencia internacional no ha logrado reproducir los resultados de los primeros trabajos de plicatura gástrica laparoscópica (PGL). Objetivo: Analizar los resultados a largo plazo de pacientes sometidos a PGL. Materiales y Método: Estudio prospectivo y descriptivo, se incluyeron pacientes obesos adultos que cumplieron criterios universales para cirugía bariátrica. Se registraron datos epidemiológicos, comorbilidades, tiempo operatorio, estadía hospitalaria, porcentaje de pérdida de exceso de IMC (\% PEIMC), complicaciones posoperatorias y resolución de comorbilidades. El seguimiento se efectuó con controles periódicos anuales hasta el año 2020. Resultados: Se inició la selección de pacientes durante el año 2010. Se realizaron 26 intervenciones desde enero de 2011 hasta mayo de 2012. Todas las pacientes fueron de género femenino. El IMC preoperatorio promedio fue $38,8 \mathrm{~kg} / \mathrm{m}^{2}$ (DS 3,8$)$. El $\%$ PEIMC promedio al año, 3 años y 9 años de posoperado, fue $62,2 \%$ (DS 27,1), 40,2\% (DS 24,5) y $28 \%$ (DS 31,9), respectivamente. Las complicaciones, basadas en la clasificación de Clavien-Dindo (CD), durante los primeros 30 días de posoperatorio fueron 21 pacientes con tipo I, 1 con tipo II, y 2 pacientes con complicaciones tipo IVa. A los 9 años de posoperado, 9 pacientes presentaban efectos adversos tipo I. No hubo mortalidad. Hasta los 3 años hubo corrección de comorbilidades. Se objetivaron 3 pacientes diabéticas al final del estudio, 2 de ellas previamente sanas. Conclusiones: El \% PEIMC a largo plazo fue insuficiente. El porcentaje de complicaciones es mayor que en otras técnicas. No recomendamos la realización de la PGL.

Palabras clave: plicatura gástrica; cirugía bariátrica; obesidad. 


\section{Introducción}

La obesidad es uno de los principales problemas de salud a nivel mundial. Datos aportados por la Organización Mundial de la Salud (OMS) han estimado que más de 1.900 millones de adultos en el mundo presentan sobrepeso ${ }^{1,2}$. En Chile el porcentaje se encuentra en $71 \% \%^{3}$.

Es sabido que existe correlación entre un incremento en la obesidad y el aumento de mortalidad por enfermedades cardiovasculares y neoplásicas. Debido a lo anterior, la prevención de la obesidad es uno de los principales objetivos en los sistemas de salud, pero no es suficiente. La cirugía bariátrica ha demostrado su eficacia en resolver la obesidad y comorbilidades, con evidencia de su impacto positivo $^{4-9}$.

Estas operaciones son clasificadas como restrictivas, malabsortivas y sus combinaciones ${ }^{10-12}$. Sin embargo, sus costos y complicaciones han causado la búsqueda de procedimientos alternativos. Se han introducido una variedad de procedimientos, incluida la plicatura gástrica, con resultados controverti$\operatorname{dos}^{13-32}$. La PGL fue innicialmente aplicada el año 2007 en pacientes por Talebpour ${ }^{16}$, con resultados buenos, pero estudios posteriores no han logrado reproducirlos $18,20,21,23,24,26-31$.

El objetivo de este trabajo es analizar la experiencia en un seguimiento de 9 años de posoperado de pacientes sometidos a PGL.

\section{Materiales y Método}

Se diseñó un protocolo prospectivo y descriptivo el año 2010, para el manejo de pacientes con obesidad con la técnica de PGL, en el servicio de cirugía del Hospital del Salvador. Este protocolo se basó en las guías de indicación de cirugía bariátrica recomendadas por la International Federation for the Surgery of Obesity and Metabolic Disorders (IFSO) y la National Institutes of Health de Estados Unidos (NIH) Conference ${ }^{33}$, todo debidamente autorizado y con datos respaldados ante el comité de ética de nuestra institución. El estudio comenzó el año 2010 con la selección de los pacientes, y los procedimientos se realizaron entre enero 2011 hasta mayo 2012.

\section{Criterios de inclusión}

Paciente con edades entre 18 y 65 años, con IMC $\geq 30\left(\mathrm{~kg} / \mathrm{m}^{2}\right)$ y con comorbilidades, o un IMC $\geq 40$ $\left(\mathrm{kg} / \mathrm{m}^{2}\right)$ con o sin comorbilidades.

\section{Criterios de exclusión}

Pacientes con cirugía gástrica previa, úlcera gástrica e infarto agudo al miocardio últimos 6 meses, patología oncológica activa, patologías psiquiátricas y/o adicciones que impiden cooperación, embarazo, patología de base grave, trastorno compulsivo alimentario, padres con cáncer gástrico.

Todos los pacientes fueron evaluados por un equipo multidisciplinario. Se realizaron exámenes preoperatorios generales, metabólicos, electrocardiograma, endoscopía digestiva alta, radiografía de tórax y ecografía abdominal, no se realizó estudio radiológico para control de filtraciones en el posoperatorio inmediato ${ }^{34}$. Todo lo anterior según las recomendaciones de las guías previamente descritas. Los procedimientos se realizaron por el mismo equipo quirúrgico.

El diagnóstico de hipertensión arterial (HTA), dislipidemia, diabetes, prediabetes y síndrome de resistencia a la insulina (SRI), se basaron en las guías clínicas correspondientes (Tabla 1), o fueron diagnósticos realizados en pacientes con el debido tratamiento previo a la evaluación inicial preoperatoria, y cuyos valores para determinada patología, se encontraban dentro de rangos normales. En cuanto a esteatosis hepática, se objetivó mediante ecografía abdominal previa y/o en el intraoperatorio. Los problemas osteoarticulares se definieron como la presencia de dolor articular crónico o patología articular relacionadas con el exceso de peso. El reflujo gastroesofágico (RGE) se objetivó como el antecedente de devolución crónica de contenido estomacal a esófago.

La resolución de comorbilidades fue definida como un valor normal de la comorbilidad estudiada, asociada a la ausencia de medicamento para

\section{Tabla 1. Criterios diagnósticos para comorbilidades}

\footnotetext{
Dislipidemia:

Colesterol total $>\mathrm{o}=$ a $200 \mathrm{mg} / \mathrm{dl} ; \mathrm{HDL}<\mathrm{o}=50 \mathrm{mg} / \mathrm{dl}$ en mujeres, 40 en hombres; Triglicéridos $>\mathrm{o}=150 \mathrm{mg} / \mathrm{dl} ; \mathrm{LDL}>\mathrm{o}=70 \mathrm{mg} / \mathrm{dl}$ (ACC/AHA 2018)

Diabetes:

Glicemia en ayuno $>\mathrm{o}=126 \mathrm{mg} / \mathrm{dl}$, glicemia poscarga $2 \mathrm{~h}>\mathrm{o}=200 \mathrm{mg} / \mathrm{dl}$, glicemia aleatoria $>\mathrm{o}=200 \mathrm{mg} / \mathrm{dl}$ asociado a síntomas, HbGlicosilada $>0=6,5 \%($ ADA 2020)

Prediabetes: Glicemia poscarga $>0=140$ a $199 \mathrm{mg} / \mathrm{dl}$, glicemia en ayuno $>_{\mathrm{o}}=100$ a $125 \mathrm{mg} / \mathrm{dl}$, Hbglicosilada $>\mathrm{o}=5,7$ a 6,4\%. (ADA 2020)

Síndrome de resistencia a la insulina:

HOMA $>2,5$, insulina basal $>12 \mathrm{ui} / \mathrm{ml}$, insulina poscarga $2 \mathrm{~h}>60 \mathrm{ui} / \mathrm{ml} *$

Cuadro de definiciones obtenido a partir de las guías clínicas de la American College of Cardiology (ACC)/American Heart Association (AHA) 2018, y de la American Diabetes Association (ADA) 2020. * Referencias 39 y 40.
} 


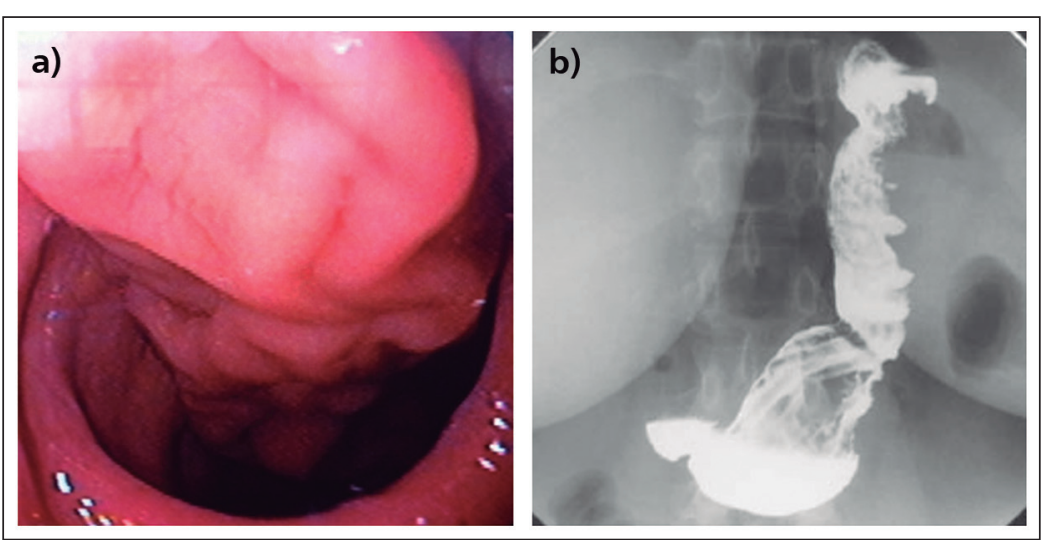

Figura 1. a) Imagen endoscópica de plicatura gástrica laparoscópica. b) Imagen radiológica al mes de posoperado y con una reducción del $80 \%$ del volumen gástrico.

Tabla 2. Datos preoperatorios

\begin{tabular}{|c|c|c|c|c|c|}
\hline Paciente & $\begin{array}{c}\text { Edad } \\
\text { operatoria } \\
\text { (años) }\end{array}$ & $\begin{array}{c}\text { Talla } \\
\text { (metros) }\end{array}$ & $\begin{array}{r}\text { Peso } \\
\text { (kg) }\end{array}$ & $\begin{array}{c}\text { Índice de } \\
\text { masa corporal } \\
\left(\mathrm{kg} / \mathrm{mts}^{2}\right)\end{array}$ & $\begin{array}{c}\text { Antecedente de } \\
\text { colecistectomía } \\
\text { previa }\end{array}$ \\
\hline 1 & 44 & 1,58 & 112 & 44,9 & 0 \\
\hline 2 & 34 & 1,60 & 90 & 35,2 & 0 \\
\hline 3 & 41 & 1,63 & 112,5 & 42,3 & 1 \\
\hline 4 & 39 & 1,47 & 78 & 36,1 & 0 \\
\hline 5 & 45 & 1,60 & 103 & 40,2 & 1 \\
\hline 6 & 36 & 1,55 & 108 & 45 & 0 \\
\hline 7 & 52 & 1,48 & 86 & 39,3 & 0 \\
\hline 8 & 59 & 1,60 & 114 & 44,5 & 0 \\
\hline 9 & 52 & 1,63 & 118 & 44,4 & 1 \\
\hline 10 & 44 & 1,62 & 100 & 38,1 & 0 \\
\hline 11 & 34 & 1,67 & 122 & 43,8 & 0 \\
\hline 12 & 39 & 1,62 & 99,5 & 37,9 & 0 \\
\hline 13 & 36 & 1,71 & 106 & 36,3 & 0 \\
\hline 14 & 38 & 1,65 & 102 & 37,5 & 0 \\
\hline 15 & 38 & 1,70 & 109 & 37,7 & 1 \\
\hline 16 & 56 & 1,57 & 91,5 & 37,1 & 0 \\
\hline 17 & 43 & 1,60 & 102 & 39,8 & 0 \\
\hline 18 & 48 & 1,62 & 109 & 41,5 & 1 \\
\hline 19 & 45 & 1,70 & 114 & 39,5 & 1 \\
\hline 20 & 30 & 1,66 & 87 & 31,6 & 0 \\
\hline 21 & 41 & 1,58 & 95 & 38,1 & 0 \\
\hline 22 & 45 & 1,56 & 90 & 37 & 0 \\
\hline 23 & 32 & 1,60 & 100 & 39,1 & 0 \\
\hline 24 & 50 & 1,60 & 98 & 38,3 & 0 \\
\hline 25 & 46 & 1,65 & 85 & 31,2 & 0 \\
\hline 26 & 32 & 1,53 & 78 & 33,3 & 0 \\
\hline
\end{tabular}

Todas las pacientes fueron de género femenino, con un promedio de edad de 42,3 años (DS 7,6) al momento de operarse. tal patología, en el control de al menos 1 año de posoperado.

Técnica quirúrgica, previamente informada ${ }^{19}:$ Se inicia con la esqueletización del estómago desde 6 $\mathrm{cm}$ del píloro, hasta el ángulo de His con línea de sección a $2 \mathrm{~cm}$ por fuera del borde de la curvatura mayor. Bajo calibración con sonda número 42 y apoyo endoscópico simultáneo (en los primeros casos) se procede a una invaginación de la curvatura mayor gástrica con puntos separados de seda $2 / 0$. Luego una segunda invaginación con punto continuo de prolene 2/0 (Figura 1).

Los pacientes fueron seguidos mediante un programa con controles presenciales, encuestas y evaluaciones telefónicas. Los datos incluyeron información demográfica, historia médica, comorbilidades, tiempo operatorio, seguimiento del peso, y complicaciones posoperatorias.

Las complicaciones y efectos adversos posoperatorios fueron divididos según la clasificación de Clavien-Dindo $(\mathrm{CD})^{35}$.

Para análisis estadístico se utilizaron los programas Microsoft Excel ${ }^{\circledR} 16.38$ y Stata/SE ${ }^{\circledR}$ 15.1. Se utilizó el test de Mann Whitney para evaluar diferencias estadísticas entre los subgrupos de pacientes $(\mathrm{p}<0,05)$.

\section{Resultados}

Se realizaron las evaluaciones preoperatorias habituales para cirugía bariátrica, con los criterios de exclusión previamente descritos. Fueron incorporadas 26 pacientes en el estudio. Éstas fueron intervenidas desde enero de 2011 hasta mayo de 2012. El promedio de edad operatorio fue 42,3 años (DS 7,6). Todos los casos incluidos fueron mujeres. El promedio de peso e IMC preoperatorio fue 100,4 $\mathrm{kgs}$ (DS 12,1) y $38,8 \mathrm{~kg} / \mathrm{m}^{2}$ (DS 3,8), respectivamente. 6 pacientes presentaban el antecedente de colecistectomía previa (Tabla 2). El tiempo quirúrgico promedio fue 94,9 minutos (DS 24,8). El promedio de hospitalización fue 5,5 días (rango 1-74 días).

Al año de posoperado, el 100\% de las pacientes presentaron al menos un control posoperatorio. Entre el primer y tercer año de posoperado, el 80,8\% presentaron uno o más controles. A los 9 años de posoperatorio, se logró un $57,7 \%$ de pacientes evaluadas.

El máximo porcentaje de pérdida de exceso de IMC (\% PEIMC) se ubicó al año de posoperado, con un promedio de $62,2 \%$ (DS 27,1), 65,4\% registró control en ese momento. A los 3 años fue 40,2\% (DS 24,5), con 76,9\% de pacientes controladas. 
Finalmente, a los 9 años luego de la cirugía, con $57,7 \%$ de pacientes evaluadas, se registró un promedio de $28 \%$ de \% PEIMC (DS 31,9). Es importante destacar que, durante el seguimiento, 2 presentaron embarazo cuyo proceso fue exitoso (Figura 2).

En cuanto al análisis de las complicaciones posoperatorias, según la clasificación de $\mathrm{CD}$, durante el período comprendido dentro de los 30 primeros días de posoperado, 21, 1 y 2 pacientes presentaron complicaciones tipo I, II y IVa, respectivamente.

Las tipo I fueron náuseas con/sin vómitos, RGE y dolor abdominal. En algunos casos cedieron con el tiempo. Además, una paciente presentó infarto esplénico de manejo conservador, otra una infección de herida operatoria y una con seroma.

Hubo una paciente con cuatro complicaciones tipo II: hemorragia digestiva alta autolimitada, nutrición parenteral completa por desnutrición calórico-proteica, delirium y reacción adversa a medicamento.

Dos pacientes presentaron complicaciones tipo IVa. Una de ellas presentó una fístula gástrica asociada a una colección intraabdominal. La otra paciente desarrolló una trombosis porto-mesentérica 11 días posterior al procedimiento, en la que se realizó una resección del segmento intestinal comprometido. Evolucionó con tromboembolismo pulmonar y neumonía asociada a ventilación mecánica. Estudios confirmaron que padecía de una trombofilia, la cual fue manejada con anticoagulantes.

Durante los 60 días siguientes se observaron 13 pacientes con complicaciones tipo I y 1 con tipo II. Las tipo I consistieron en náuseas con/sin vómi- tos, RGE y dolor abdominal. Una misma paciente presentó un trastorno de deglución leve y sepsis por catéter venoso central. Lo anterior evidencia la resolución de las comorbilidades más complejas sucedidas en el primer período.

Evaluando los efectos adversos según la clasificación de CD, luego de 90 días hasta los 3 años de posoperada. 15 pacientes presentaron eventos tipo I y 6 tipo IIIb. En las tipo I, se evidenciaron náuseas con/sin vómitos, RGE y dolor abdominal. De las 6 pacientes con tipo IIIb, una paciente requirió un bypass gástrico revisional resectivo, debido a un RGE importante. Un caso con una hernia gástrica, que requirió una gastrectomía vertical tubular laparoscópica revisional. A una se le realizó una endoscopía digestiva alta de urgencia producto de una hemorragia digestiva. Por último, 3 pacientes fueron sometidas a una reparación de hernia incisional. Estos datos muestran la persistencia en algunas pacientes de eventos de menor gravedad en el tiempo, y la aparición de otros que fueron resueltas.

A los 9 años de posoperatorio, 9 pacientes presentaron efectos adversos tipo I: 3 con náuseas con/ sin vómitos recurrentes, 5 RGE y 4 con dolor abdominal (Figuras 3 y 4).

Al analizar las comorbilidades, del total de pacientes al inicio del estudio, 53,9\% presentaban HTA $(\mathrm{n}=14), 80,8 \%$ dislipidemia $(\mathrm{n}=21), 3,9 \%$ diabetes $(n=1), 38,5 \%$ prediabetes $(n=10) y$ $76,9 \%$ SRI $(n=20)$. Además de $38,5 \%$ problemas osteoarticulares $(n=10), 65,4 \%$ esteatosis hepática $(\mathrm{n}=17)$ y $3,9 \% \operatorname{RGE}(\mathrm{n}=1)$. A los 3 años de seguimiento el porcentaje de pacientes recuperadas,

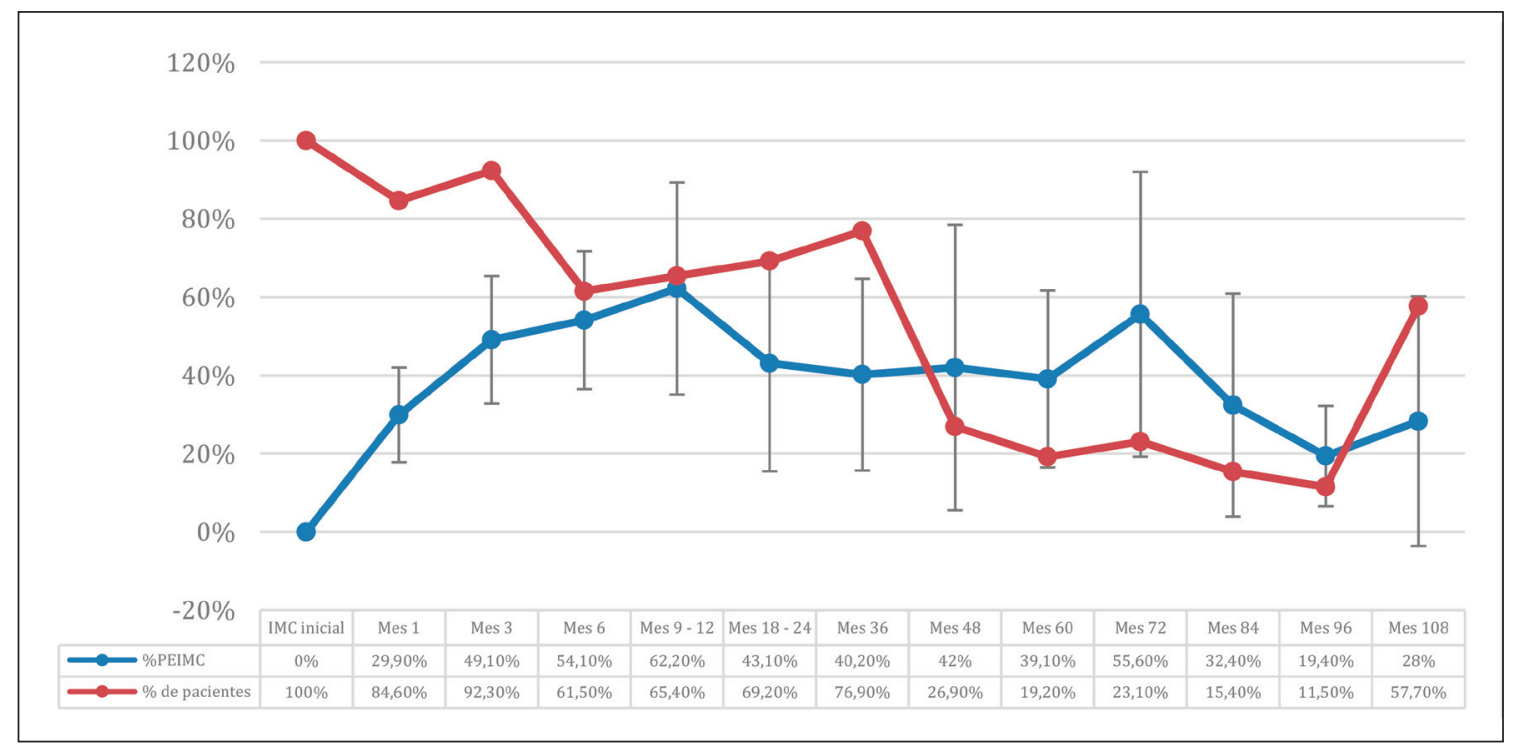

Figura 2. Gráfico que ilustra la evolución del Porcentaje de Pérdida de Exceso de IMC (\% PEIMC) comparando el porcentaje de pacientes evaluados, durante el tiempo. 


\section{ARTÍCULO ORIGINAL}

Figura 3. Ilustra el número de complicaciones y efectos adversos posoperatorios según la clasificación de Clavien Dindo, en los siguientes períodos: durante los primeros 30 días de posoperatorio, entre 31 y 90 días, entre 91 días y 3 años, y a los 9 años de posoperado. *Náuseas y/o vómitos contabilizada como evento único.
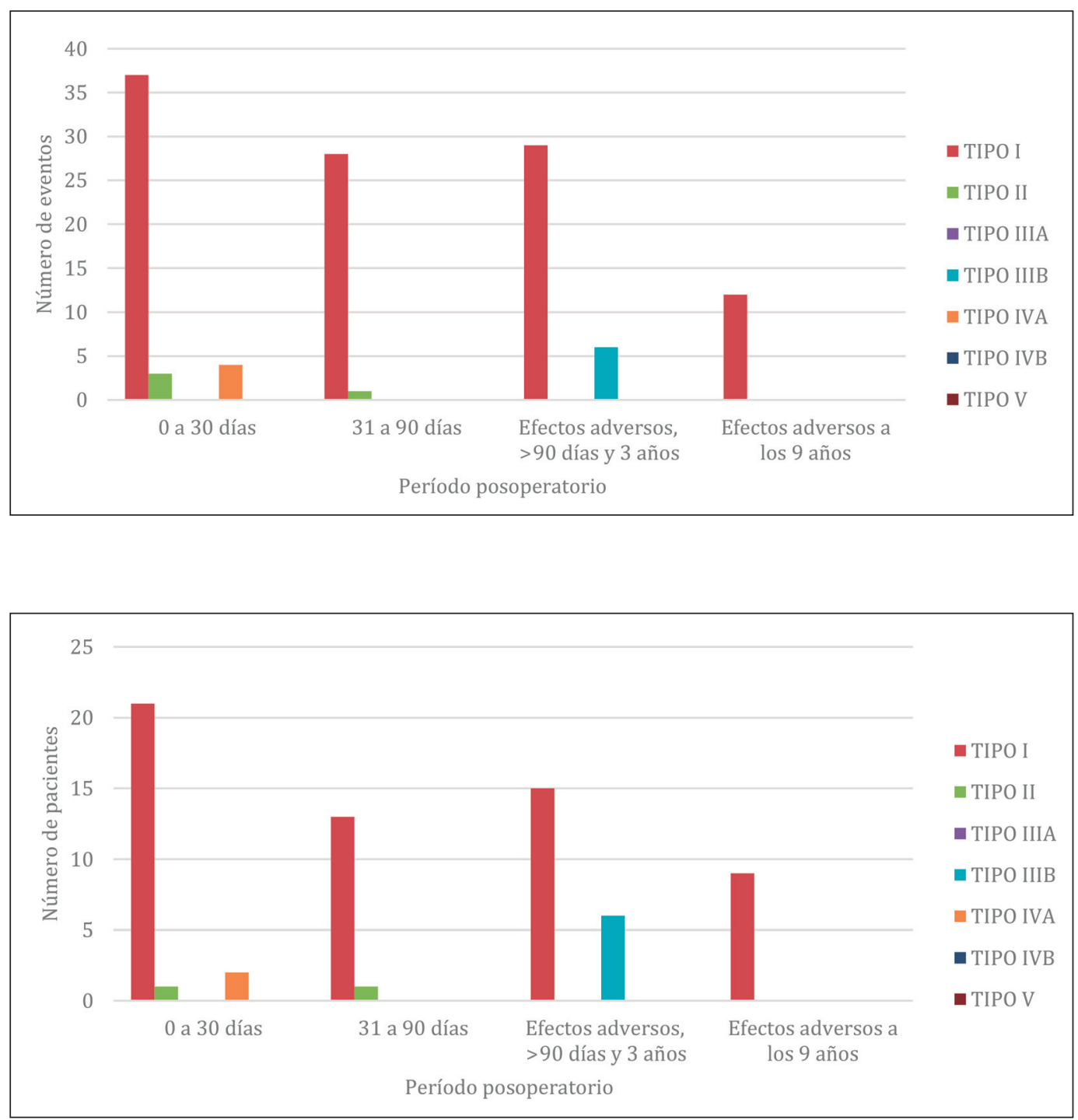

Figura 4. Ilustra el número de pacientes con complicaciones y efectos adversos posoperatorios según la clasificación de Clavien Dindo, en los siguientes períodos: durante los primeros 30 días de posoperatorio, entre 31 y 90 días, entre 91 días y 3 años, y a los 9 años de posoperado. *Náuseas y/o vómitos contabilizada como evento único. fue $26,9 \%$ para HTA $(\mathrm{n}=7), 30,8 \%$ dislipidemia $(\mathrm{n}=8), 3,9 \%$ diabetes $(\mathrm{n}=1), 23 \%$ prediabetes $(n=6)$ y $30,8 \%$ problemas osteoarticulares $(n=8)$. Con respecto a la aparición de nuevas patologías previamente inexistentes, a los 3 años de posoperatorio, se registró tanto para diabetes, prediabetes, HTA y dislipidemia, un caso para cada una, respectivamente. Tanto para para el SRI y esteatosis hepática, los datos fueron insuficientes para sacar conclusiones (Tabla 3 ).

A los 9 años de posoperadas, 7 pacientes fueron objetivadas con problemas osteoarticulares, en 5 de ellas se había registrado resolución de estos a los 3 años y volvieron a presentarlos al final del estudio. En el mismo control posoperatorio, 3 de nuestras pacientes presentaban diabetes mellitus, 2 de ellas previamente sanas (Tabla 3).

Una de las 3 pacientes diabéticas al final del estudio presentó un infarto agudo al miocardio durante su evolución. Hubo 3 pacientes que desarrollaron enfermedades neoplásicas malignas, las cuales fueron resueltas de manera exitosa. Se destaca la diferencia del \% PEIMC entre estos 6 últimos casos $(29,4 \%)$ y el promedio del grupo (40,2\%) al tercer año. Debido al número reducido no se pudieron establecer diferencias estadísticas (Tabla 4). Cuatro pacientes fueron colecistectomizadas posterior a la PGL.

No hay diferencia estadísticamente significativa entre las pacientes con obesidad inicial tipo I y II, en comparación a las pacientes con obesidad tipo 
Tabla 3. Tabla de número de casos de pacientes con comorbilidades y su seguimiento

\begin{tabular}{|c|c|c|c|c|c|}
\hline & $\begin{array}{l}\text { Prevalencia } \\
\text { inicial } \\
\text { (n) }\end{array}$ & $\begin{array}{l}\text { Resolución } \\
\text { a } 3 \text { años } \\
\text { (n) }\end{array}$ & $\begin{array}{c}\text { Nuevos casos a } 3 \text { años } \\
\text { de posoperatorio } \\
\text { (n) }\end{array}$ & $\begin{array}{c}\text { Resolución a } 9 \text { años } \\
\text { de posoperatorio } \\
\text { (n) }\end{array}$ & $\begin{array}{c}\text { Nuevos casos a } 9 \text { años } \\
\text { de posoperatorio } \\
\text { (n) }\end{array}$ \\
\hline$\%$ de seguimiento & $100 \%$ & $76,9 \%$ & $76,9 \%$ & $57,7 \%$ & $57,7 \%$ \\
\hline Hipertensión arterial & 14 & 7 & 1 & 2 & 0 \\
\hline Dislipidemia & 21 & 8 & 1 & 8 & 0 \\
\hline Prediabetes & 10 & 6 & 1 & - & - \\
\hline Resistencia a la insulina & 20 & - & - & - & - \\
\hline Problemas osteoarticulares & 10 & 8 & 0 & 1 & $7 *$ \\
\hline Esteatosis hepática & 17 & - & - & - & - \\
\hline
\end{tabular}

Se observa la prevalencia inicial de comorbilidades en número de pacientes y su evolución en el tiempo. Se comparó la prevalencia inicial con los datos obtenidos a los 3 y 9 años de estudio. *Una paciente presentó resolución de diabetes mellitus a los 3 años, al final del estudio la presentó nuevamente. En 5 pacientes se observó resolución de problemas osteoarticulares a los 3 años y volvieron a registrarse al final del estudio.

Tabla 4. Eventos neoplásicos malignos, accidentes cardiovasculares y diabetes mellitus a 9 años de posoperatorio versus \% PEIMC

\begin{tabular}{|c|c|c|c|}
\hline Paciente & $\begin{array}{l}\text { Evento durante los } 9 \text { años de } \\
\text { posoperatorio }\end{array}$ & $\%$ PEIMC & $\begin{array}{l}\text { Año de control } \\
\text { posoperatorio }\end{array}$ \\
\hline 1 & Nevo melanocítico & $51,3 \%$ & $3 .^{e r}$ año \\
\hline 2 & Recidiva cáncer de tiroides & $22 \%$ & $3 .^{\text {er }}$ año \\
\hline 3 & Cáncer de cuello uterino & $29,5 \%$ & $4 .^{\circ}$ año \\
\hline 4 & Infarto agudo al miocardio $+\mathrm{DM}$ & $31 \%$ & $3 .^{\text {er }}$ año \\
\hline 5 & $\mathrm{DM}$ & $33,6 \%$ & $3 .^{\text {er }}$ año \\
\hline 6 & $\mathrm{DM}$ & $8,7 \%$ & 3. ${ }^{\text {er }}$ año \\
\hline Promedio \% PEIMC pacientes 1 al 6 & - & $29,4 \%$ & $3 .^{\text {er }}$ y $4 .^{\circ}$ año \\
\hline Promedio de $\%$ PEIMC 3 er $^{\text {er }}$ ão posoperatorio ( $76,9 \%$ de controlados) & - & $40,2 \%$ & $3 .^{e r}$ año \\
\hline Promedio \% PEIMC $4 .^{\circ}$ año posoperatorio ( $26,9 \%$ de controlados) & - & $42 \%$ & $4 .^{\circ}$ año \\
\hline Promedio \% PEIMC $9 .^{\circ}$ año posoperatorio $(57,7 \%$ de controlados $)$ & - & $28 \%$ & $9 .^{\circ}$ año \\
\hline
\end{tabular}

Se observa una descripción de eventos neoplásicos malignos, accidentes cardiovasculares y pacientes con diabetes mellitus, sucedidos durante los 9 años de posoperatorio. Se compara el \% PEIMC de este grupo de pacientes y el promedio total, al tercer, cuarto y noveno año de posoperatorio. DM: diabetes mellitus.

III, y sus resultados en \% PEIMC al año, 3 años y 9 años de posoperatorio. Tampoco hubo diferencias estadísticamente significativas entre el IMC inicial y la presencia o ausencias de complicaciones y/o efectos adversos quirúrgicos, en los diferentes periodos hasta los 3 años de posoperado. A los 9 años de posoperatorio, se objetivó una diferencia entre el IMC inicial, y la presencia de efectos adversos. A mayor IMC inicial, mayor posibilidad de presentar efectos adversos a los 9 años $(p=0,02)$.

\section{Discusión}

El valor más importante de este trabajo es el seguimiento a 9 años de posoperatorio de PGL. Evidenciamos un \% PEIMC en rango bueno hasta el año, para posteriormente disminuir a rango de fracaso terapéutico, además de una mala corrección de comorbilidades y mayores complicaciones posoperatorias, en comparación a otras cirugías bariátricas. 
Es sabido que ciertas comorbilidades presentan una mejoría y/o resolución en pacientes después de un procedimiento bariátrico. Más aún, estudios poblacionales han evidenciado que la cirugía disminuye la incidencia de enfermedades cardiovasculares, cáncer y la mortalidad global ${ }^{4-9}$. Los costos y complicaciones de estas cirugías han estimulado la búsqueda de otras técnicas. De esta manera emergió la PGL, la cual tendría un menor costo debido al no usar sutura mecánica, y menores complicaciones debido a la ausencia de resección gástrica y de anastomosis.

Algunos autores preconizaron la PGL con resultados favorables, pero con incompleto seguimiento a largo plazo. Otros publicaron series con un importante número de complicaciones, y reporte de casos con eventos adversos, presentando una postura más cautelosa ${ }^{13-31}$. Iniciamos nuestro estudio durante el año 2010, y publicamos nuestro primer caso con resultados promisorios el año $2011^{13}$.

El tener un grupo de pacientes compuesto de mujeres representa lo publicado en estudios previos, en que son mujeres las que en mayor proporción se someten a una cirugía bariátrica ${ }^{6-8}$.

Durante el primer año nuestro estudio presentó cifras similares, y posterior a este, cifras superiores en seguimiento, que otras publicaciones de PGL ${ }^{25,31}$. Este fue inferior a los datos arrojados por trabajos escandinavos de cirugía bariátrica ${ }^{6,7}$. Mantuvimos un contacto proactivo con nuestras pacientes, lo que nos lleva a plantear a futuro una forma de trabajar similar.

En cuanto a indicador de calidad, el \% PEIMC en la PGL al año de posoperado, se ubica en rango bueno, con un $62,2 \%$, mejor que en el estudio publicado por Alice Albanece $(46,5 \%)^{31,36}$. Pero, existe una clara tendencia a la disminución de este buen resultado luego del año de posoperado, teniendo con el transcurso del tiempo un promedio oscilante entre $20 \%$ y $45 \%$ de \% PEIMC, clasificándose como fracaso terapéutico.

Uno de los objetivos principales de esta técnica fue la de disminuir las complicaciones, pero evidenciamos lo contrario. Nuestros resultados coinciden con la literatura ${ }^{18,20,21,23,24,26-31}$. Lo que resultó en un mayor costo tanto económico como de calidad de vida de nuestras pacientes.

El hecho de encontrar una relación entre el mayor IMC inicial y la mayor posibilidad de presentar efectos adversos a largo plazo, con diferencia estadística significativa, nos permite concluir que los pacientes con obesidad tipo 1 son los que más se beneficiaron del procedimiento. Al tener un seguimiento de un 58\% al noveno año de poso- peratorio, reconocemos que existe una muestra parcial del total, sin posibilidad de obtener más conclusiones.

Evidenciamos una clara resolución de las comorbilidades hasta los 3 años, pero con porcentajes inferiores a otros procedimientos, aún más, observamos la aparición de patologías en nuestros pacientes previamente inexistentes ${ }^{9}$. Además, la aparición durante la evolución de nuestra cohorte, de enfermedades neoplásicas y cardiovasculares, nos hace concluir que este procedimiento no logró la prevención lograda por la cirugía bariátrica para estas patologías descrita en otros estudios ${ }^{6,7}$.

Durante los últimos años se ha difundido la técnica de la plicatura gástrica endoscópica, también llamada gastroplastía endoscópica, con resultados a largo plazo aun no informados, la que no está exenta de complicaciones y con un \% PEIMC no superior al $50 \%$ al año ${ }^{32}$. Este estudio tiene un valor comparativo, ya que al ser la PGL una técnica similar, se puede inferir que los resultados a largo plazo de la plicatura endoscópica pudiesen ser similares y deberían ser considerados como un importante patrón de referencia.

A lo largo de la historia de la cirugía bariátrica se han utilizado técnicas de plastía con sutura mecánica, sin sección gástrica, con resultados no satisfactorios, debido a la tendencia del restablecimiento de la anatomía normal. Lo anterior es aplicable para esta técnica ${ }^{37}$.

Además, una desventaja que tiene la plicatura gástrica es el impedimento de la observación endoscópica adecuada de la zona. Esto tiene una importancia mayor en Chile, donde la tasa de mortalidad por cáncer gástrico es importante ${ }^{38}$.

\section{Conclusión}

Los resultados a largo plazo de la PGL tienen un índice mayor de complicaciones que otras técnicas bariátricas tradicionales, no corrige comorbilidades y no logra producir una reducción de peso efectiva. A largo plazo, a diferencia de otros procedimientos, no logra evitar el desarrollo de patologías tales como neoplasias malignas y accidentes cardiovasculares. Estos resultados pueden ser un referente importante a considerar a la hora de evaluar la plicatura gástrica endoscópica.

Por lo tanto, no recomendamos el uso de la PGL como un procedimiento bariátrico equivalente a los otras técnicas quirúrgicas que actualmente se emplean. 


\section{Responsabilidades éticas}

\section{Autorizado por el Comité de Ética Local}

Protección de personas y animales. Los autores declaran que para esta investigación no se han realizado experimentos en seres humanos ni en animales.
Confidencialidad de los datos. Los autores declaran que en este artículo no aparecen datos de pacientes.

Agradecimientos: Srta. Claudia López.

Conflictos de interés: no hay.

\section{Bibliografía}

1. World Health Organization. The Global Health Observatory. Prevalence of overweight among adults, BMI $>=25$ (crude estimate) (\%). Publicado el 28 de septiembre de 2017 (consultado el 15 de agosto de 2020). Disponible en: https:// www.who.int/data/gho/data/indicators/ indicator-details/GHO/prevalenceof-overweight-among-adults-bmigreaterequal-25-(crude-estimate)-(-)

2. World Health Organization. Centro de prensa, notas descriptivas. Obesidad y sobrepeso. Publicado el primero de abril del 2020 (consultado el 15 de agosto de 2020). Disponible en: https://www. who.int/es/news-room/fact-sheets/detail/ obesity-and-overweight.

3. Ministerio de Salud, Gobierno de Chile. Departamento de Epidemiología. División de Planificación Sanitaria. Subsecretaría de Salud Pública. Santiago de Chile. Encuesta nacional de salud 2016-2017. Publicado en noviembre 2017 (consultado el 15 agosto de 2020). Disponible en: https://www.minsal.cl/ wp-content/uploads/2017/11/ENS-201617_PRIMEROS-RESULTADOS.pdf.

4. Eeg-Olofsson K, Cederholm J, Nilsson P, Zethelius B, Nunez L, Gudbjörnsdóttir S, et al. Risk of cardiovascular disease and mortality in overweight and obese patients with type 2 diabetes: an observational study in 13,087 patients, Diabetologia 2009;52:65-73.

5. Pan S, Johnson K, Ugnat A, Wen S, Mao Y, and Canadian Cancer Registries Epidemiology Research Group. Association of Obesity and Cancer Risk in Canada. Am J Epidemiol. 2004;159:25968.

6. Sjöström L, Narbro K, Sjöström C, Karason K, Larsson B, Wedel H, et al. Effects of Bariatric Surgery on Mortality in Swedish Obese Subjects. N Engl J Med. 2007;357:741-52.

7. Sjostrom L. Review of the key results from the Swedish Obese Subjects (SOS) trial - a prospective controlled intervention study of bariatric surgery. J Intern Med. 2013;273;219-34.

8. Tao W, Santoni G, Von Euler-Chelpin M, Ljung R, Lynge E, Pukkala E, et al. Cancer Risk After Bariatric Surgery in a Cohort Study from the Five Nordic Countries. Obes Surg. published online 13 june 2020.

9. English W, Williams D.B. Metabolic and Bariatric Surgery: A Viable Treatment Option for Obesity. Prog Cardiovasc Dis. 2018;61:253-69.

10. DeMaria E. Bariatric Surgery for Morbid Obesity. N Engl J Med. 2007;356:217683.

11. Sumithran P, Prendergast L, Delbridge E, Purcell K, Shulkes A, Kriketos A, Proietto J. Long-Term Persistence of Hormonal Adaptations to Weight Loss. N Engl J Med. 2011;365:1597-604.

12. Korner J, Leibel R. To Eat or Not to EatHow the Gut Talks to the Brain. N Engl J Med. 2003;349:926-8.

13. Fusco P, Poggetti R, Younes R, Fontes B, Birolini D. Evaluation of Gastric Greater Curvature Invagination for Weight Loss in Rats. Obes Surg. 2006;16:172-7.

14. Fusco P, Poggetti R, Younes R, Fontes B, Birolini D. Comparison of Anterior Gastric Wall and Greater Gastric Curvature Invaginations for Weight Loss in Rats. Obes Surg. 2007;17:1340-5.

15. Sales C. Surset gástrico de Sales: una alternativa para cirugía bariátrica restrictiva. Rev Colomb Cir. 2008;23:1315.

16. Talebpour M, Amoli B. Laparoscopic total gastric vertical plication in morbid obesity. J Laparoendosc Adv Surg Tech A. 2007; 17:793-8.
17. Ramos A, Galvao M, Galvao G, Evangelista L, Marins J, Ferraz A. Laparoscopic Greater Curvature Plication: Initial Results of an Alternative Restrictive Bariatric Procedure. Obes Surg. 2010;20:913-8.

18. Brethauer S, Harris J, Kroh M, Schauer P. Laparoscopic gastric plication for treatment of severe obesity. Surgery for Obesity and Related Diseases 2011;7:15 22.

19. Contreras J, Villao D, Bravo J, Núñez J, Camacho J, Czwiklitzer G, et al. Laparoscopic gastric plication, a new bariatric procedure: report of one case. Rev Chil Cir. 2011;63:399-403.

20. Shen D, Ye H, Wang Y, Ji Y, Zhan X, Zhu J, Li W. Comparison of short-term outcomes between laparoscopic greater curvature plication and laparoscopic sleeve gastrectomy. Surg Endosc. 2013;27:2768-74.

21. Pujol J, García A, Casajoana A, Secanella L, Vicens A, Masdevall C. Gastroplastia tubular plicada, una nueva técnica para el tratamiento de la obesidad mórbida. Cir Esp. 2011;89:356-61

22. Kourkoulos M, Giorgakis E, Kokkinos C, Mavromatis T, Griniatsos J, Nikiteas N, et al. Laparoscopic Gastric Plication for the Treatment of Morbid Obesity: A Review. Minim Invasive Surg. 2012;2012:696348. doi: $10.1155 / 2012 / 696348$.

23. Atlas H, Yazbek T, Garneau P, Safa N, Denis R. Is There a Future for Laparoscopic Gastric Greater Curvature Plication (LGGCP)? A Review of 44 Patients. Obes Surg. 2013;23:1397403.

24. Niazi M, Maleki A, Talebpour M. ShortTerm Outcomes of Laparoscopic Gastric Plication in Morbidly Obese Patients: Importance of Postoperative Follow-up. Obes Surg. 2013;23:87-92. 
25. Talebpour M, Motamedi S, Talebpour A, Vahidi $H$. Twelve years experience of laparoscopic gastric plication in morbid obesity: development of the technique and patient outcomes. Ann Surg Innov Res. 22;6:7:1-16.

26. Taha O. Efficacy of Laparoscopic Greater Curvature Plication for Weight Loss and Type 2 Diabetes: 1-Year Follow-Up. Obes Surg. 2012;22:1629-32.

27. Hii M, Clarke N, Hopkins G. Gastrogastric herniation: an unusual complication following greater curve plication for the treatment of morbid obesity. Ann R Coll Surg Engl. 2012;94:e76-e78.

28. Watkins B. Case report. Gastric compartment syndrome: an unusual complication of gastric plication surgery. Surg Obes Relat Dis. 2012;8:e80-1.

29. Tsang A, Jain V. Case report. Pitfalls of bariatric tourism: a complication of gastric plication. Surg Obes Relat Dis. 2012;8:e77-9.

30. Skrekas G, Antiochos K, Stafyla V.
Laparoscopic Gastric Greater Curvature Plication: Results and Complications in a Series of 135 Patients. Obes Surg. 2011;21:1657-63.

31. Albanese A, Prevedello L, Verdi D, Nitti D, Vettor R, Foletto M. Laparoscopic Gastric Plication: An Emerging Bariatric Procedure with High Surgical Revision Rate. Bariatr Surg Pract Patient Care 2015;10:93-8.

32. Lopez-Nava G, Galvao M P, BautistaCastaño I, Jimenez-Baños A, FernandezCorbelle J P. Endoscopic Sleeve Gastroplasty: How I Do It? Obes Surg. 2015;25:1534-8.

33. NIH conference. Gastrointestinal surgery for severe obesity. Consensus Development Conference Panel. Ann Intern Med. 1991;115:956-61.

34. Medina F, Miranda-Merchak A, Martínez A, Sánchez F, Bravo S, Contreras JE, et al. Usefulness of upper gastrointestinal series to detect leaks in the early postoperative period of bariatric surgery. Rev Med Chile 2016;144:451-5.
35. Dindo D, Demartines N, Clavien P-A. Classification of surgical complications: a new proposal with evaluation in a cohort of 6336 patients and results of a survey. Ann Surg. 2004;240:205-13.

36. Larrad A, Sánchez-Cabezudo C. Indicadores de calidad en cirugía bariátrica y criterios de éxito a largo plazo. Cir Esp. 2004;75:301-4.

37. Csendes A, Korn O, Burdiles P. Historia de la cirugía bariátrica en Chile (19862005). Rev Chil Cir. 2006;58:386-9.

38. Csendes A, Figueroa M. Situación del cáncer gástrico en el mundo y en Chile. Rev Chil Cir. 2017;69:502-7.

39. Acosta AM, Escalona M, Maiz A, Pollak F, Leighton F. Determination of the insulin resistance index by the Homeostasis Model Assessment in a population of Metropolitan Region in Chile. Rev Med Chile 2002;130:1227-31.

40. Carrasco F, Galgani JE, Reyes M. Síndrome de resistencia a la insulina. Estudio y manejo. Rev Med Clin Condes 2013;24:827-37. 